\title{
Resonance production and decay in pion induced collisions with HADES
}

\author{
Federico Scozzi ${ }^{1,2, a}$ for the HADES collaboration \\ ${ }^{1}$ TU Darmstadt, Germany \\ ${ }^{2}$ IPN Orsay, France
}

\begin{abstract}
The main goal of the High Acceptance Di-Electron experiment (HADES) at GSI is the study of hadronic matter in the $1-3.5 \mathrm{GeV} /$ nucleon incident energy range. HADES results on $e^{+} e^{-}$production in proton-nucleus reactions and in nucleus-nucleus collisions demonstrate a strong enhancement of the dilepton yield relative to a reference spectrum obtained from elementary nucleon-nucleon reactions. These observations point to a strong modification of the in-medium $\rho$ spectral function driven by the coupling of the $\rho$ to baryon-resonance hole states. However, to scrutinize this conjecture, a precise study of the role of the $\rho$ meson in electromagnetic baryon-resonance transitions in the time-like region is mandatory. In order to perform this study, HADES has started a dedicated pion-nucleon programme. For the first time a combined measurement of hadronic and dielectron final states have been performed in $\pi^{-}-N$ reactions at four different pion beam momenta $(0.656,0.69,0.748$ and $0.8 \mathrm{GeV} / \mathrm{c})$. First results on exclusive channels with one pion $\pi^{-}-p$ and two pions $\left(n \pi^{+} \pi^{-}, p \pi^{-} \pi^{0}\right)$ in the final state, which are currently analysed within the partial wave analysis (PWA) framework of the Bonn-Gatchina group, are discussed. Finally first results for the dielectron analysis will be shown.
\end{abstract}

\section{Introduction}

The High Acceptance Di-Electron spectrometer (HADES) [1] operating at GSI was designed with the purpose of studying the hadronic matter using rare and penetrating probes, such as dielectrons or strange particles in an energy range where moderate temperatures $(\mathrm{T}<80 \mathrm{MeV})$ and large densities (up to 3 times the normal nuclear matter density) are reached [1]. At the same time an experimental programme, using pp, "quasifree" np and proton-nucleus reactions, was carried out in order to check what are the modifications coming from the medium effects. Starting from the work of Brown and Rho [2], the role of the $\rho$ meson, that in vacuum can be interpreted as two pion excitation, that decays mainly into a $\pi^{+} \pi^{-}$pair, was pointed out to be crucial for in-medium effects. In fact the life time of this meson is so short that it decays inside the medium created in heavy-ion interactions. Therefore using the decay into an electron-positron pair, that don't interact strongly in the medium, it is possible to study the characteristics of $\rho$. The observable - chosen to check the different theoretical scenarios is the invariant mass, that is linked to the spectral function. The challenge is also experimental since the branching ratio of the $\rho$ into dileptons is very small $\left(4.72 \times 10^{-5}\right)$ and hadronic background is very large.

\footnotetext{
ae-mail: f.scozzi@gsi.de
} 
Although the mechanism for the $\rho$ production is different at high and at low energy, the experimental measurements, ranging form HADES energies up to RHIC (see contribution of P. Salabura's to this conference [3]), can be interpreted by means of the modification of the in-medium $\rho$ propagator due to a strong coupling in the pion loop $\pi \Delta N^{-1}$ or a direct $\rho$ meson- $N^{-1} N^{*}$ excitation, where $N^{*}$ is a baryonic resonance. In particular, the Rapp-Wambach scenario [4] with an in-medium broadening is confirmed by various experiments.

Due to the coupling of the $\rho$ meson to baryonic resonances, its decay into an $e^{+} e^{-}$pair is related to the electromagnetic structure of baryonic transitions, parameterized using electromagnetic form factors which are functions of the four-momentum transfer squared $q^{2}$. Two kinematical regions can be accessed. The space-like region, corresponding to negative values of $q^{2}$, is studied by means of electron proton scattering reactions. A large amount of data was collected expecially by the CLAS experiment at JLab [5]. The time-like region, corresponding to positive values of $q^{2}$, can be studied by means of annihilation reactions, for which few data are available, and of the $\mathrm{N}^{*} \rightarrow \mathrm{Ne}^{+} e^{-}$Dalitz decays, that allow to study the region of $q^{2}$ smaller than the difference between the squared resonance mass and the nucleon mass. Moreover the real photon limit $\left(q^{2}=0\right)$ is known experimentally using $\gamma p$ interactions.

The decay rates of a baryonic resonance into a dilepton pair and a nucleon can be written using three (e.g. electric, magnetic and Coulomb) form factors for spin 3/2 (or larger) resonances or 2 form factors for spin $1 / 2$ resonances. A model for the electromagnetic transition form factors is needed. The more simple approach is a "QED point-like" $R \gamma^{*}$ model [6], that uses constant form factors calculated at real photon point $\left(q^{2}=0\right)$. Another approach is the Vector Meson Dominance (VMD) model, introduced by J.J. Sakurai [7], that assumes that the electromagnetic current is mediated by ground state vector mesons, which yields monopole form factors; however it was proved that the standard VMD model overestimates the branching ratios of the nucleon resonances for radiative decay. To circumvent this problem, extended VMD [8], in which the excited states of the vector mesons $\rho^{\prime}, \rho^{\prime \prime}, \ldots$ are taken into account for the description of the transition form factors of the nucleon resonances, was introduced. Constituent quark models for transition form factors used for the space like region have also been extended recently to the time like region [9], [10].

We present a preliminary analysis of data measured by HADES in the reactions $\pi^{-} p \rightarrow n \pi^{+} \pi^{-}, \pi^{-} p$ $\rightarrow \pi^{0} \pi^{-}, \pi^{-} p \rightarrow X e^{+} e^{-}$. The combination of hadronic and electromagnetic channels has been chosen to shed light on the role of the $\rho$ meson in the electromagnetic structure of baryonic transitions, and the validity of the VMD.

\section{The HADES experiment}

HADES is placed at GSI Helmholtzzentrum für Schwerionenforschung in Darmstadt. It is a fixedtarget experiment with a large angular acceptance (almost full coverage in the azimuthal angle and between $18^{\circ}-85^{\circ}$ in the polar angle)(see Fig. 1). HADES is suitable to be used with different high intensity beams (protons, ions and pions) in the energy range of 1-3.5 GeV.

A low-mass tracking system, composed of Multiwire Drift Chambers (MDCs), and a superconducting toroidal magnet provide momentum reconstruction. Particle identification is obtained by time of flight measurements using Scintillator Time-Of-Flight (TOF) and Resistive Plate Chambers (RPC) and by energy loss measurements in MDCs. The crucial detector for the separation between lepton and hadrons is a Ring Imaging Cerenkov Detector (RICH). In addition also an electromagnetic Pre-Shower detector can be used for improving lepton/hadron separation. 


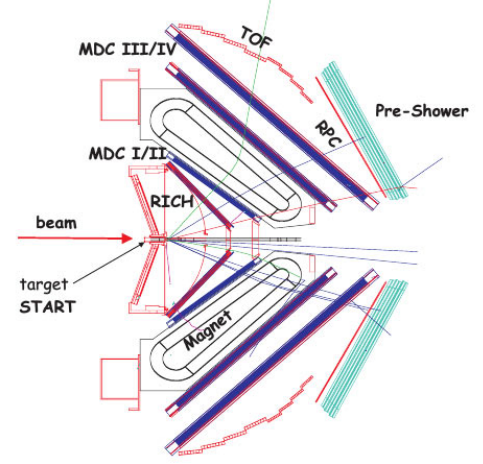

Figure 1. Schematic view of the HADES detector with indicated components.

\section{Pion beam experiment}

The idea of a pion beam facility at SIS18 was developed at the beginning of the nineties and the first commissioning run test happened already in 1999 [11]. The secondary pion beam is generated by a primary $N_{2}$ beam provided by the SIS 18 synchrotron with an intensity of $8 \times 10^{10}$ ions/spill, that interacts with a thick beryllium target. The pions are transported to the HADES target, located $33 \mathrm{~m}$ downstream of the production point, by a beam line composed of a system of 9 quadrupole and 2 dipole magnets (Fig. 2). A transmission of $56 \%$ is achieved for a given central momentum with a distribution that can be well approximated by a Gaussian distribution with $\sigma=1.5 \%$. The pion intensity distribution at the exit of the pion beam line reaches a maximum of about $10^{6}$ pions/spill at $\mathrm{p}=1.0 \mathrm{GeV} / \mathrm{c}$ and decreases to about half of this value at $\mathrm{p}=0.7 \mathrm{GeV} / \mathrm{c}$. The measurement of the momentum of the pion beam particles is furnished by a dedicated tracking system, called CERBEROS [12]. It is composed by two double-sided silicon detectors with a thickness of $0.3 \mathrm{~mm}$. They cover an area of $10 \times 10 \mathrm{~cm}^{2}$ and are segmented into 128 horizontal and vertical strips. The location of the two detectors in the beam line can be seen in the Fig. 2. Combining the measurement of positions $(\mathrm{X}, \mathrm{Y})$ provided by the two detectors, it is possible to reconstruct the momentum of each pion beam particle with a resolution of about $0.3 \%$. The contamination of the $\pi^{-}$beam is mainly due to electrons arising from conversion of the two photons from $\pi^{0}$ decays in the production target. A contamination of $0.8 \%, 3.2 \%$ and $9.8 \%$ is calculated for reference momenta of the pion beam line of respectively $1.7 \mathrm{GeV} / \mathrm{c}, 1.0 \mathrm{GeV} / \mathrm{c}$ and $0.7 \mathrm{GeV} / \mathrm{c}$.

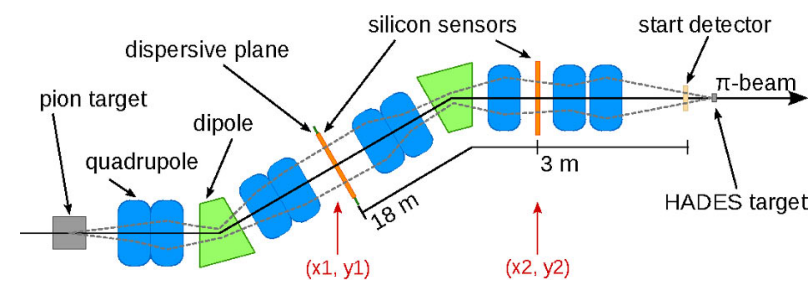

Figure 2. Schematic view of the pion beam line. 
The data discussed in this work were taken in summer 2014 with two weeks of beam on target in total. The first run was realized in order to study the strangeness production in cold matter in pioninduced reactions on light $\left({ }^{12} C\right)$ and heavy $\left({ }^{74} \mathrm{~W}\right)$ nuclei at a pion beam momentum of $1.7 \mathrm{GeV} / \mathrm{c}$. The purpose of the second run was to study $\pi^{-}-p$ reactions in the second resonance region. To reach this aim two targets were used: polyethylene $\left(\mathrm{C}_{2} \mathrm{H}_{4}\right)_{2}$ and carbon (C) with the goal to subtract events from scattering on carbon and to identify the pure contribution from scattering on protons. Data at four different pion beam momenta $(0.656,0.69,0.748$ and $0.8 \mathrm{GeV} / \mathrm{c})$ were collected with the largest statistics in the case of $0.69 \mathrm{GeV} / \mathrm{c}$ momentum, chosen to study dilepton production.

\section{Pion beam data analysis}

\subsection{Elastic scattering}

$\pi^{-} p$ elastic scattering was measured by HADES during the same data taking, which allows for the normalization of all other channels. To select this kind of reactions a cut on angular correlations of the reconstructed proton and pion tracks $\left(\left|\phi_{p}-\phi_{\pi}\right|<5^{\circ}\right.$ and $\left.\tan \theta_{\pi^{-}} \mathrm{x} \tan \theta_{p}>1\right)$ was applied. The squared missing mass distributions for events coming from interactions with the carbon target and with the polyethylene target are shown in Fig. 3(left panel). The carbon events (red curve) were scaled in order to match the left tail, that is distant from the peak of the elastic interaction on protons, to the distribution coming from the polyethylene target (black curve). This scaling factor can be also measured in a second way, exploiting the counting rates in START detector, to get the incoming flux and taking into account the different carbon atom density in the two targets. The two numbers are in good agreement. An additional verification of the accuracy of this scaling factor, deduced with elastic scattering, is given by the fact that it holds to all inelastic channels under analysis, as it will be shown below.
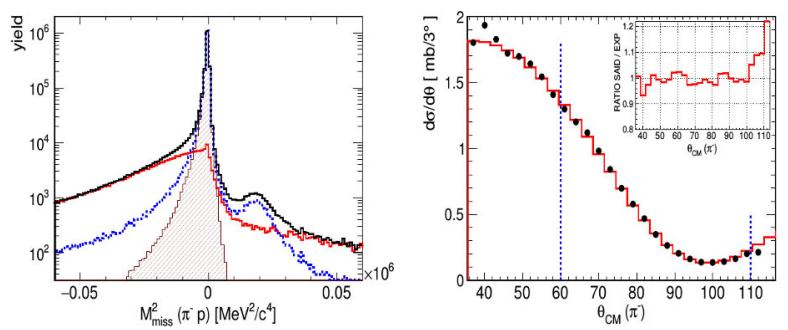

Figure 3. $\pi^{-} p \rightarrow \pi^{-} p$ elastic scattering at beam momentum $800 \mathrm{MeV} / \mathrm{c}$. Left: $\pi^{-} p$ missing mass squared with events from polyethylene target (black histogram), carbon target rescaled (red histogram) and their difference (blue dotted curve), finally, elastic scattering selection (dashed area). Right: $\theta$ angle of $\pi^{-}$in c.m.s. from $\pi^{-} p$ elastic scattering, efficiency and acceptance corrected (black dots) compared with the distribution from the SAID database within the fiducial volume $60^{\circ}<\theta_{C M}<110^{\circ}$ (limited by the vertical blue dashed lines). In the upper right corner: ratio of SAID data to reconstructed elastic $\pi^{-} p$ events.

In a second step a method to tag events measured in the polyethylene target corresponding to interactions of pions with protons was developed. First the events were grouped into bins of similar missing mass values, then for all combinations among tracks measured with the polyethylene and the carbon target those with the lowest $\chi^{2}$ were paired together. For the $\chi^{2}$ several variables were used: momentum, polar angle of the particles and the invariant mass of the pair. 
Finally, the experimental angular distributions were compared to a simulation of elastic scattering. The events were generated in the framework of the Pluto event generator [13] with angular distributions based on [14]. Then a full GEANT simulation, including the geometry and the response of the detector and using the same analysis cuts as adopted for real data was performed. Simulated elastic scattering events describe the measured data within the HADES acceptance well and they are used for the one-dimensional acceptance correction. Using the technique - described above - to subtract the quasi-elastic contribution coming from carbon, it is possible to verify that the missing mass distribution obtained in this way is in agreement with the simulation in which only $\pi^{-} p$ reactions are present.

The corrected data, as shown in Fig.3 (right panel), have been then compared to the distributions obtained from the SAID database [15] of the known differential $\pi^{-}-p$ elastic scattering in the same angular region. In this way the normalisation factors for each center-of-mass energy have been obtained. For the 4 momentum values the cross sections are: $3.01 \mathrm{mb}, 3.10 \mathrm{mb}, 3.08 \mathrm{mb}$ and $2.59 \mathrm{mb}$ in the polar angle range between $60^{\circ}$ and $110^{\circ}$ in the center of mass system.

\section{$4.2 n \pi^{-} \pi^{+}$and $p \pi^{-} \pi^{0}$ channels}
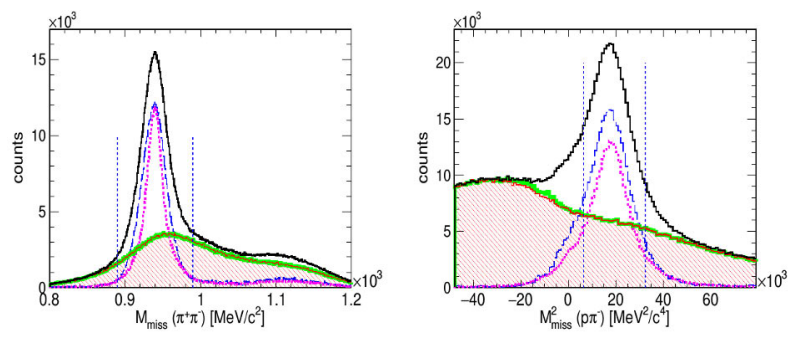

Figure 4. Results from $2 \pi$ production in $\pi^{-}$induced reactions. Black curve - events from polyethylene target, red curve and red dashed area - events from carbon target, green thick curve (behind red) - events from polyethylene target tagged as $\pi^{-} C$ interactions, blue dashed curve - events from polyethylene target tagged as $\pi^{-} p$ interactions, magenta dotted curve - signal calculated based on information on momentum measured by the pion tracker. The vertical blue dashed lines limit a window $\left( \pm 50 \mathrm{MeV} / \mathrm{c}^{2}\right.$ around the neutron missing mass) for selection of events for the partial wave analysis. Left: $\pi^{+} \pi^{-}$missing mass. Right: $p \pi^{-}$missing mass squared.

The identification of the events with one $\pi^{-}$and one $\pi^{+}$or with one $\pi^{-}$and one $p$ in the final state permits the reconstruction of the exclusive channels $\pi^{-} p \rightarrow \pi^{-} \pi^{+} n$ and $\pi^{-} p \rightarrow \pi^{-} \pi^{0} p$, respectively, exploiting the missing mass technique. In the first case one can cut in the area around the undetected neutron (see Fig 4 on the left) and in the second case in the area around the undetected neutral pion (see Fig 4 on the right). The technique described above is applied to tag the events coming from interactions with protons. The width $(1 \sigma)$ of the neutron peak in the case of the missing mass of $\pi^{-} \pi^{+}$ is of the order of $14 \mathrm{MeV} / \mathrm{c}^{2}$ using a constant beam momentum and it decreases to around $9 \mathrm{MeV} / \mathrm{c}^{2}$ using the beam momentum obtained by the pion tracker event by event. In both cases the value of the neutron mass is reproduced with a precision of $\pm 1 \mathrm{MeV} / \mathrm{c}^{2}$. In the case of the missing mass $\pi^{-} \pi^{0}$ the resolution is around $40 \%$ using a constant beam momentum value and it decreases to around $30 \%$ using the pion tracker. The position of the peak corresponding to $\pi^{0}$ is $3-4 \mathrm{MeV} / \mathrm{c}^{2}$ lower than the $\pi^{0}$ mass when using a fixed beam momentum and only $2-3 \mathrm{MeV} / \mathrm{c}^{2}$ using the pion tracker.

To be sure that the method developed for the carbon subtraction is correct, also other variables, different from the missing mass, have been used as shown in Fig. 5 for the $\cos \theta$ of the pion in the 

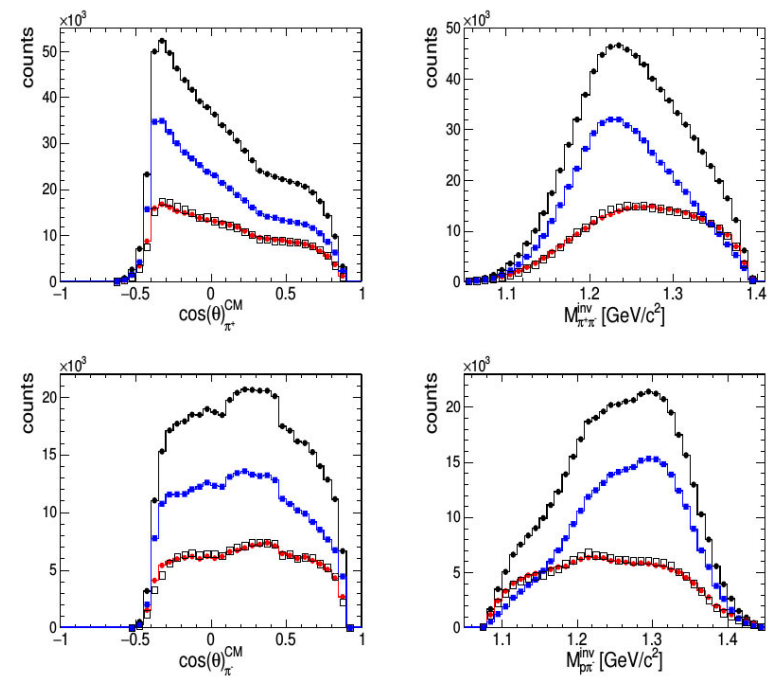

Figure 5. $\pi^{+} \pi^{-}$and $p \pi^{-}$channels measured for incident pion momenta of $0.8 \mathrm{GeV} / \mathrm{c}$. Events from polyethylene target (black histogram), events from carbon target (red histogram), events tagged as scattering on proton (blue histogram), events tagged as scattering on carbon (black open squares). In the upper row the channel $n \pi^{+} \pi^{-}$is shown. On the left $\pi^{+}$angular distribution in c.m.s.; on the right $\pi^{+} \pi^{-}$invariant mass. In the lower row the channel $p \pi^{-} \pi^{0}$ is shown. On the left $\pi^{-}$angular distribution in c.m.s; on the right $p \pi^{-}$invariant mass.

center of mass system and the invariant masses for the two channels mentioned above. The distribution obtained with events coming directly from the measurement on the carbon target is in good agreement with the distribution obtained for events measured on the polyethylene target and selected with the tag method described above.

The number of reconstructed events for the two channels at the four pion beam momenta is listed in the following table:

Table 1. Number of reconstructed events.

\begin{tabular}{ccc}
\hline $\mathrm{p}[\mathrm{GeV} / \mathrm{c}]$ & $n \pi^{-} \pi^{+}$ & $p \pi^{-} \pi^{0}$ \\
\hline 0.656 & $\sim 400000$ & $\sim 122000$ \\
0.690 & $\sim 7900000$ & $\sim 3100000$ \\
0.748 & $\sim 815000$ & $\sim 490000$ \\
0.800 & $\sim 526000$ & $\sim 368000$ \\
\hline
\end{tabular}

Considering the very large statistics and the broad phase space coverage, these data improve in a significant way the current database of di-pion channels in pion-induced reactions, that is very scarce in the second resonance region. The main analysis in this range was performed by Manley [16], [17] and it relies on around 240000 bubble chamber events $\left(n \pi^{+} \pi^{-}\right.$channel). The channel $n \pi^{0} \pi^{0}$ was later measured by the Crystal Ball collaboration [18].

These new data have been included in the multi-channel partial wave analysis framework of the Bonn-Gatchina group [19], in complement to the existing data in pion induced reactions and a large sample of meson photoproduction data. 


\section{Dilepton analysis}

\subsection{Lepton identification and purity determination}

Since dileptons are very rare probes in an environment dominated by pions, a careful study of the best compromise between efficiency and purity of the lepton sample is required. In HADES the crucial detector for lepton identification is the RICH detector. The goal in using RICH is to reconstruct a ring and to match it with a reconstructed track. The standard ring finder is based on a pattern recognition algorithm to select the good rings [1]. Recently a new approach called "backtracking" was developed [20]: a good lepton candidate in terms of velocity, momentum and energy loss is propagated back to the RICH detector in order to find a possible ring with less restrictive conditions. This approach was proved to have a higher efficiency than the standard method, keeping the capability of background rejection at a similar level [20].

HADES has developed a method to estimate the purity of the lepton sample using the standard ring finder approach, the so-called rotation RICH technique. It consists in a $60^{\circ}$ rotation (softwarewise) of the RICH detector and then in matching the tracks with the rings. In this way the correlation between the rings and the track is lost and only random matches are taken into account. From the comparison of the angular distribution between the tracks and the rings in the two cases is possible to estimate the purity of the lepton sample, defined as:

$$
\text { Purity }=1-\frac{\text { rot. } \text { RICH data }}{\text { standard data }}
$$

Table 2. Purity values.

\begin{tabular}{ccc}
\hline $\mathrm{p}[\mathrm{MeV} / \mathrm{c}]$ & $e^{+}$ & $e^{-}$ \\
\hline $\mathrm{p}<100$ & $99.7 \%$ & $99.1 \%$ \\
$100<\mathrm{p}<200$ & $99.4 \%$ & $98.7 \%$ \\
$200<\mathrm{p}<300$ & $97.7 \%$ & $93.7 \%$ \\
$300<\mathrm{p}<400$ & $97.3 \%$ & $89.0 \%$ \\
\hline
\end{tabular}

The purity depends on the momentum, especially for electrons due to negative pion contanimation, but in any case a satisfactory level (around $90 \%$ at least) is achieved for the lepton ID.

\subsection{Inclusive raw dilepton spectrum}

After identifying single leptons, one has to combine them in pairs in order to get the invariant mass spectrum, not only signal pairs are present in the sample, but also uncorrelated pairs coming from different production vertexes are present, such as leptons from two $\pi^{0}$ decays. Therefore one has to consider all possible pair combinations and an estimation of the combinatorial background (CB) due to uncorrelated pairs, is required. $\mathrm{CB}$ is evaluated by means of the same event like-sign technique: like-sign pairs $\left(N_{++}, N_{--}\right)$coming from the same events are formed and then the geometrical average is calculated: $C B=2 \sqrt{N_{++} N_{--}}$. When one of the two numbers is 0 , the arithmetic average is used. Finally the $\mathrm{CB}$ is subtracted from the unlike-sign pair sample to get the signal. In addition, to improve the background rejection, a cut on the opening angle of pairs is applied, requesting that it must be larger than $9^{\circ}$; this helps in the rejection of the CB originating from the conversion of photons from $\pi^{0}$ decay. Moreover specifical cut in the tracking procedure are used to suppress the leptons coming from close pairs. In the Fig. 6 the raw invariant mass spectrum is shown with the backtracking method for lepton ID and after applying the two cuts just mentioned. 

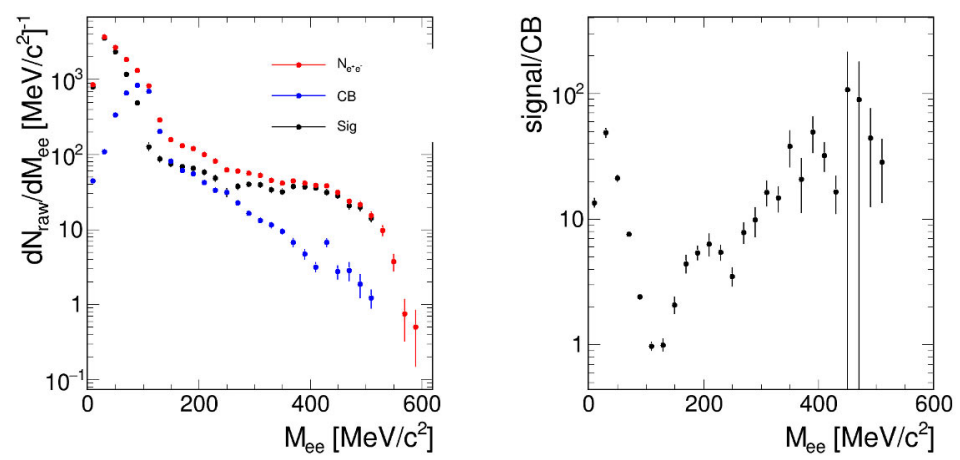

Figure 6. On the left raw inclusive invariant mass spectra using backtracking. Red solid circles show all $e^{+} e^{-}$ combinations, blue solid circles show $\mathrm{CB}$ estimated using geometrical mean of same-event like-sign pairs, black solid circles show the signal Sig $=\mathrm{N}_{e^{+} e^{-}}$CB. On the right the signal to background ratio is shown.

The number of pairs with invariant mass larger than $0.140 \mathrm{GeV} / \mathrm{c}^{2}$ is $\sim 3360$ (a factor 2.8 higher than the number obtained using the standard algorithm) and the signal to background ratio is high, with a minimum around 1 for invariant mass around $0.1 \mathrm{GeV} / \mathrm{c}^{2}$ and it becomes larger (around 20) at higher invariant mass which is the region of interest for studies of the electromagnetic structure of baryon transitions.

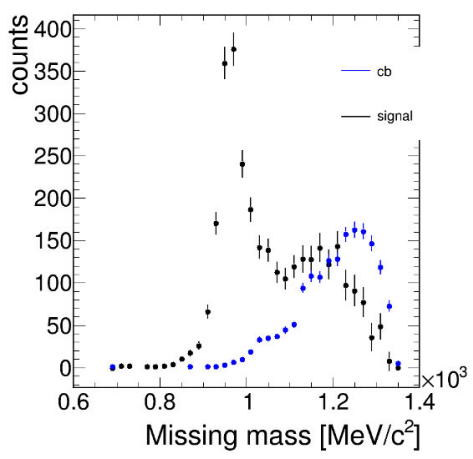

Figure 7. Missing mass spectrum for events with an $e^{+} e^{-}$pair with $M_{i n v}>140 \mathrm{MeV} / \mathrm{c}^{2}$. Blue solid circles show $\mathrm{CB}$ estimated using geometrical mean of same-event like-sign pairs, black solid circles show the signal obtained after combinatorial subtraction.

\subsection{Missing mass spectrum}

In the inclusive dilepton spectrum, several sources of electrons contribute, such as $\pi^{0}$ dalitz decay, $\eta$ dalitz decay, etc... The most interesting physics information is expected in the $\rho$ decay in $e^{+} e^{-}$, where three particles $\left(n, e^{+}\right.$and $\left.e^{-}\right)$are expected. To select this channel the missing mass technique is used 
(since HADES can not detect the neutron). The missing mass spectrum (see Fig. 7) shows a dominant peak located around the neutron mass allowing for the selection of the $e^{+} e^{-}$final state.

\section{Conclusions}

Recently, the HADES collaboration performed an experiment using the GSI pion beam at a momentum of about $0.7 \mathrm{GeV} / \mathrm{c}$ and polyethylene and carbon targets. A large amount of data was collected for the hadronic channels $n \pi^{-} \pi^{+}$and $p \pi^{-} \pi^{0}$ with a broad acceptance, allowing for the measurement of multidifferential distributions. The events measured in the polyethylene target can be tagged as corresponding either to an interaction with a proton or with a carbon nucleus, allowing for a very precise selection of $\pi-p$ interactions. The on-going PWA, which includes these new data and the existing pion and photon beam data base, will provide updated information on $2 \pi$ couplings in the $\mathrm{N}(1520)$ region. Di-electon production in pion-induced reactions in the second resonance region was measured for the first time. The collected statistics should permit to test different theoretical predictions and, in combination with the two pion production, to get information on the role of $\rho$ meson in time-like electromagnetic transitions of the produce baryon resonances.

\section{Acknowledgements}

The HADES collaboration gratefully acknowledges the support by the grants PTDC/FIS/113339/2009 LIP Coimbra, NCN grant 2013/10/M/ST2/00042 SIP JUC Cracow, Helmholtz Alliance HA216/EMMI GSI Darmstadt, VH-NG-823, Helmholtz Alliance HA216/EMMI TU Darmstadt, 283286, 05P12CRGHE HZDR Dresden, BMBF 06MT7180 TU München, Garching BMBF:05P12RGGHM JLU Giessen, Giessen UCY/3411-23100, University Cyprus CNRS/IN2P3, CNRS/IN2P3 (France), MSMT LG 12007, AS CR M100481202, GACR 13-06759S NPI AS CR, Rez, EU Contract No. HP3-283286.

\section{References}

[1] G. Agakichiev et al. (HADES collaboration), Eur. Phys. J. A 41, 243 (2009)

[2] G.E. Brown and M. Rho, Phys. Rev. Lett. 66, 2720 (1991)

[3] P. Salabura, "HADES experiment probing baryonic matter at SIS18 : overview of results", this conference

[4] R. Rapp and J. Wambach, Eur. Phys. J. A 6, 415 (1999)

[5] I.G. Aznauryan et al., Phys. Rev. C 80, 055203 (2009)

[6] M. Zetenyi and G. Wolf, Phys. Rev. C 67, 044002 (2003)

[7] J.J. Sakurai, Phys. Rev. Lett. 22, 981 (1969)

[8] M. I. Krivoruchenko, B. V. Martemyanov, A. Faessler, and C. Fuchs, Annals Phys. 296, 299 (2002)

[9] G. Ramalho, M.T. Pena, Phys.Rev. D 85, 113014 (2012)

[10] G. Ramalho and M.T. Peña, arXiv:1610.08788 [nucl-th]

[11] J. Diaz et al., Nucl. Instrum. Meth. A 478, 511 (2002)

[12] R. Lalik et al., 2013 IEEE Nuclear Science Symposium and Medical Imaging Conference (2013), DOI: 10.1109/NSSMIC.2013.6829440

[13] I. Fröhlich et al., PoS ACAT2007 076 (2007) 
[14] A.D. Brody et al., Phys. Rev. D 3, 2619 (1971)

[15] SAID database: http://gwdac.phys.gwu.edu/

[16] D.M. Manley, R.A. Arndt, Y. Goradia, V.L. Teplitz, Phys. Rev. D 30, 904 (1984)

[17] D.M. Manley and E.M. Saleski, Phys. Rev. D 45, 4002 (1992)

[18] S. Prakhov et al. (Crystal Ball), Phys. Rev. C 69, 045202 (2004)

[19] A. Anisovich, E. Klempt, A.V. Sarantsev and U. Thoma, Eur. Phys. J. A 24, 111 (2005)

[20] P. Sellheim (HADES collaboration), J. Phys. Conf. Ser. 599, 012027 (2015) 